\title{
CERTAIN COLLECTIONS OF ARCS IN $E^{8}$
}

\author{
B. J. BALL
}

1. Introduction. In considering upper semicontinuous decompositions of $E^{3}$, it is sometimes useful to know whether a given collection of continua can be transformed, by a homeomorphism of $E^{3}$ onto itself, into another collection which is simpler in some respects; for example, a collection of straight line intervals might be transformed into a collection of vertical intervals, or a collection of arcs into a collection of straight line intervals. It might also be useful to know conditions under which such a transformation can be effected by means of a particular type of homeomorphism of $E^{3}$ onto itself.

In this paper, the following questions of this type will be considered. Suppose $\alpha$ and $\beta$ are horizontal planes and $G$ is a continuous collection of mutually exclusive arcs, each of which is irreducible from $\alpha$ to $\beta$ and no one of which contains two points of any horizontal plane, such that the sum of the elements of $G$ is compact and intersects $\alpha$ in a totally disconnected set. Under what conditions is there a homeomorphism of $E^{3}$ onto itself which takes each element of $G$ onto a vertical interval and does not change the $z$-coordinate of any point?

It is shown, with the aid of certain results due to Bing [1] and Fort [5], that such a transformation is not always possible, even when the elements of $G$ are straight line intervals. The following condition is found to be necessary and sufficient for the existence of such a transformation (see $\$ 3$ for definitions of unfamiliar terms): For every positive number $\epsilon$ there exists a finite set $K_{1}, K_{2}, \cdots, K_{n}$ of topological cylinders with bases on $\alpha$ and $\beta$ such that (1) the solid cylinders determined by $K_{1}, K_{2}, \cdots, K_{n}$ are mutually exclusive, (2) each arc of $G$ is enclosed by some $K_{i}$ and (3) each $K_{i}$ has horizontal diameter less than $\epsilon$.

2. Examples. The decomposition given by Bing in [1] can be modified so that the collection of nondegenerate elements is of the type considered above. If there were a homeomorphism of $E^{3}$ onto itself carrying these arcs onto vertical intervals, then by $[2$, Theorem 5 ], the decomposition space would be homeomorphic to $E^{3}$; since this is not the case, there is no such homeomorphism.

A stronger example is furnished by Fort's modification [5] of Bing's example. This modification can be carried out in such a way

Presented to the Society, January 28, 1958; received by the editors January 15 , 1959. 
that there exist four horizontal planes $\alpha, \beta, \gamma, \delta$ with $\alpha$ above $\beta$, $\beta$ above $\gamma$, and $\gamma$ above $\delta$, such that each nondegenerate element of the decomposition is the sum of three intervals $g_{1}, g_{2}, g_{3}$ with end points on $\alpha$ and $\beta, \beta$ and $\gamma$, and $\gamma$ and $\delta$, respectively. Let $G$ denote the collection of all nondegenerate elements of this decomposition and let $G_{1}, G_{2}$ and $G_{3}$ denote, respectively, the collection of all intervals lying in an element of $G$ and having end points on $\alpha$ and $\beta$, the collection of all such intervals having end points on $\beta$ and $\gamma$, and the collection of all those with end points on $\gamma$ and $\delta$. Suppose there is a homeomorphism of $E^{3}$ onto itself which does not change the $z$-coordinate of any point and which takes each element of $G_{1}$ onto a vertical interval. Then there is a homeomorphism $f_{1}$ of $E^{3}$ onto itself which is fixed on $\beta$ and on all points below $\beta$ and which takes each element of $G_{1}$ onto a vertical interval. From the symmetry of the construction of $G$, it follows that there is also a homeomorphism $f_{3}$ of $E^{3}$ onto itself which is fixed on $\gamma$ and all points above $\gamma$ and which takes each element of $G_{3}$ onto a vertical interval. Then $f_{3} f_{1}$ is a homeomorphism of $E^{3}$ onto itself which is fixed on $\beta, \gamma$ and all points between $\beta$ and $\gamma$ and which takes each element of $G_{1}+G_{3}$ onto a vertical interval. The proof of the main theorem below shows that there is a homeomorphism $f_{2}$ of $E^{3}$ onto itself which is fixed on $\gamma$ and all points below $\gamma$, does not change the $z$-coordinate of any point, takes each element of $G_{2}$ onto a vertical interval, and is such that if $p$ and $q$ are two points above $\beta$ and on the same vertical line, then $f_{2}(p)$ and $f_{2}(q)$ are on the same vertical line. The transformation $f_{2} f_{3} f_{1}$ is a homeomorphism of $E^{3}$ onto itself which takes each element of $G$ onto a vertical interval. But this is impossible since it implies, as before, that the decomposition space is homeomorphic to $E^{3}$. Hence the elements of $G_{1}$ cannot be transformed into a collection of vertical intervals by a homeomorphism of $E^{3}$ onto itself which does not change the $z$-coordinate of any point.

It is perhaps worth noting that if $G_{1}^{\prime}$ is the decomposition of $E^{3}$ whose only nondegenerate elements are the elements of $G_{1}$, then the decomposition space of $G_{1}^{\prime}$ is homeomorphic to $E^{3}$. This is a consequence of the following theorem, which is essentially proved in [2]. If $G$ is a monotone upper semicontinuous decomposition of $E^{3}$ such that (1) the set of nondegenerate elements of $G$ is 0 -dimensional in the decomposition space and (2) for every positive number $\epsilon$ and every open set $U$ containing the sum of the nondegenerate elements of $G$, there is a homeomorphism of $E^{3}$ onto itself which is fixed on $E^{3}-U$ and which takes each element of $G$ into a set of diameter less than $\epsilon$, then the decomposition space is homeomorphic to $E^{3}$. 
3. Definitions. If $G$ is a collection of sets, then $G^{*}$ will denote the sum of the elements of $G$; the collection $G$ is said to fill up a point set $M$ if $G^{*}=M$.

A subset $K$ of $E^{3}$ will be called a topological cylinder provided there exist two parallel planes $\alpha_{0}$ and $\alpha_{1}$ and a continuous collection $G$ of mutually exclusive arcs filling up $K$ such that (1) each element of $G$ is irreducible from $\alpha_{0}$ to $\alpha_{1}$, (2) no element of $G$ contains two points of any plane parallel to $\alpha_{0}$, and (3) $\alpha_{0} \cdot K$ and $\alpha_{1} \cdot K$ are simple closed curves. The planar disks bounded by $\alpha_{0} \cdot K$ and $\alpha_{1} \cdot K$ will be called the bases of $K$ and the collection $G$ will be called a set of generators for $K$. A topological cylinder plus its bases will be called a closed topological cylinder and a closed topological cylinder plus its interior will be called a solid topological cylinder.

If $K$ is a topological cylinder with bases on the planes $\alpha_{0}$ and $\alpha_{1}$, then $K$ is said to enclose a point set $M$ provided that (1) each point of $M$ lies either between the planes $\alpha_{0}$ and $\alpha_{1}$ or else on one of those planes and (2) if $\alpha$ is a plane parallel to $\alpha_{0}$ or $\alpha_{1}$ and intersecting $M$, then the simple closed curve $\alpha \cdot K$ encloses $\alpha \cdot M$ (i.e., $\alpha \cdot M$ is a subset of the bounded component of $\alpha-\alpha \cdot K)$.

If $K$ is a topological cylinder with horizontal bases, then $\max (\operatorname{dia}(\alpha \cdot K)), \alpha$ a horizontal plane, will be called the horizontal diameter of $K$.

4. Lemma 1. Suppose $A$ is the annulus bounded by the unit circle $C_{1}$ and the circle $C_{2}$ with center $O$ and radius 2 , and $G$ is a collection of mutually exclusive arcs filling $u p A$ such that each arc of $G$ has one end point on $C_{1}$ and the other on $C_{2}$ and no arc of $G$ contains two points of any circle with center $O$. Then there exists an isotopy $\left\{F_{t}\right\}, 0 \leqq t \leqq 1$, such that (1) for each $t, F_{t}$ is a homeomorphism of $A$ onto itself which does not change the distance from $O$ of any point, (2) $F_{0}$ is the identity on $A$ and (3) $F_{1}$ is a homeomorphism which takes each element of $G$ into an interval lying on a line through $O$.

Proof. Let $g_{0}$ be an element of $G$. There is a continuous function $\phi(r), 1 \leqq r \leqq 2$, such that $g_{0}$ has the polar coordinate equation $\theta=\phi(r)$. For each $t$ in $[0,1 / 2]$ and each point $(r, \theta)$ of $A$, let $F_{t}^{1}(r, \theta)$ $=(r, \theta-2 t \cdot \phi(r))$. Then $\left\{F_{t}^{1}\right\}, 0 \leqq t \leqq 1 / 2$, is an isotopy on $A$ and $F_{0}^{1}$ is the identity. If $(r, \theta) \in g_{0}$, then $F_{1 / 2}^{1}(r, \theta)=(r, 0)$, so $F_{1 / 2}^{1}$ takes $g_{0}$ onto the interval with end points $(1,0)$ and $(2,0)$.

Let $g_{0}^{\prime}=F_{1 / 2}^{1}\left(g_{0}\right)$ and let $G^{\prime}$ denote the collection of all images under $F_{1 / 2}^{1}$ of elements of $G$. For each point $p$ of $A$, let $\theta(p)$ be the smallest non-negative polar angle for $p$ and let $\pi(p)$ denote the point of intersection of $C_{1}$ and the arc of $G^{\prime}$ containing $p$. For each $t$ in $[1 / 2,1]$ 
and each point $p=(r, \theta)$ of $A$, let $F_{t}^{2}(p)=(r, 2(1-t) \cdot \theta(p)+(2 t-1)$ $\cdot \theta(\pi(p)))$.

Since $\pi(p)$ is continuous on $A$ and $\theta(p)$ is continuous on $A-g_{0}^{\prime}$, $F_{t}^{2}$ is continuous on $A-g_{0}^{\prime}$. By a direct argument, it can be shown that $F_{t}^{2}$ is also continuous at each point of $g_{0}^{\prime}$, so it is continuous on all of $A$. From the fact that if $\theta\left(p_{1}\right)<\theta\left(p_{2}\right)$, then $\theta\left(\pi\left(p_{1}\right)\right)<\theta\left(\pi\left(p_{2}\right)\right)$, it follows that $F_{t}^{2}$ is $1-1$ and hence is a homeomorphism. It is easily verified that $\left\{F_{t}^{2}\right\}, 1 / 2 \leqq t \leqq 1$, is an isotopy. Clearly $F_{1 / 2}^{2}$ is the identity on $A$, and since for each $p$ in $A, \theta(\pi(p))$ is a polar angle for $F_{1}^{2}(p)$ and $\pi(p)$ is constant on any element of $G^{\prime}, F_{1}^{2}$ takes each element of $G^{\prime}$ into an interval lying on a line through $O$.

For each $t$ in $[0,1 / 2]$, let $F_{t}=F_{t}^{1}$ and for each $t$ in $[1 / 2,1]$, let $F_{\imath}=F_{t}^{2} F_{1 / 2}^{1}$. Then $\left\{F_{t}\right\}, 0 \leqq t \leqq 1$, is an isotopy satisfying the desired conditions.

Lemma 2. Suppose $K_{1}$ and $K_{2}$ are right circular cylinders with horizontal bases such that $K_{2}$ encloses $K_{1}$. For $i=1,2$, let $G_{i}$ be a set of generators for $K_{i}$ and let $U_{i}$ denote the interior of the closed cylinder determined by $K_{i}$. Then there exists a continuous collection $G$ of mutuclly exclusive arcs filling up the closure of $U_{2}-U_{1}$ such that no element of $G$ contains two points of any horizontal plane and such that $G_{1}+G_{2} \subset G$.

Proof. Suppose $K_{i}, i=1,2$, is represented in cylindrical coordinates by the equations $r=i, 0 \leqq z \leqq 1$. It follows from Lemma 1 that there is an isotopy $\left\{F_{t}^{1}\right\}, 1 \leqq t \leqq 3 / 2$, such that (1) for each $t$ in $[1,3 / 2], F_{t}^{1}$ is a homeomorphism of $K_{1}$ onto itself which does not change the $z$-coordinate of any point, (2) $F_{3 / 2}^{1}$ is the identity on $K_{1}$ and (3) $F_{1}^{1}$ takes each element of $G_{1}$ onto a vertical interval. Similarly, there exists an isotopy $\left\{F_{t}^{2}\right\}, 3 / 2 \leqq t \leqq 2$, such that for each $t$ in $[3 / 2,2], F_{t}^{2}$ is a homeomorphism of $K_{2}$ onto itself which does not change the $z$-coordinate of any point, (2) $F_{3 / 2}^{2}$ is the identity on $K_{2}$ and (3) $F_{2}^{2}$ takes each element of $G_{2}^{\prime}$ onto a vertical interval.

Let $M=\mathrm{Cl}\left(U_{2}-U_{1}\right)$ and for each point $p=(r, \theta, z)$ of $M$, let $F(p)$ be the point $\left(r, \theta^{\prime}, z\right)$, where $\theta^{\prime}$ is such that if $r \leqq 3 / 2, F_{r}^{1}(1, \theta, z)$ $=\left(1, \theta^{\prime}, z\right)$ and if $r \geqq 3 / 2, F_{r}^{2}(2, \theta, z)=\left(2, \theta^{\prime}, z\right)$. Then $F$ is a homeomorphism of $M$ onto itself which does not change the $z$-coordinate of any point. Since $F$ agrees with $F_{1}^{1}$ on $K_{1}$ and with $F_{2}^{2}$ on $K_{2}$, it takes each element of $G_{1}+G_{2}$ onto a vertical interval.

Let $G^{\prime}$ denote the collection of all vertical intervals lying in $M$ and having one end point on $\alpha_{0}$ and the other on $\alpha_{1}$ and let $G$ denote the collection of all images under $F^{-1}$ of elements of $G^{\prime}$. Then $G$ is a collection of mutually exclusive arcs fillng up $M$ and satisfying the desired conditions. 
Lemma 3. Suppose $K_{0}, K_{1}, K_{2}, \cdots, K_{n}$ are topological cylinders with bases on the horizontal planes $\alpha_{0}$ and $\alpha_{1}$ such that $K_{0}$ encloses $K_{j}(j=1,2, \cdots, n)$ and such that no two of the solid cylinders determined by $K_{1}, K_{2}, \cdots, K_{n}$ have a point in common. If for $j=0,1,2$, $\cdots, n, G_{j}$ is a set of generators for $K_{j}$ and $U_{j}$ is the interior of the closed cylinder determined by $K_{j}$, then there is a continuous collection $G$ of mutually exclusive arcs filling up the closure of

$$
U_{0}-\left(U_{1}+U_{2}+\cdots U_{n}\right)
$$

such that (1) no element of $G$ contains two points of any horizontal plane and (2) each of $G_{0}, G_{1}, \cdots, G_{n}$ is a subcollection of $G$.

Proof. Let $K_{0}^{\prime}, \cdots, K_{n}^{\prime}$ denote right circular cylinders with bases on $\alpha_{0}$ and $\alpha_{1}$ which are related in the same way as the correspondingly lettered topological cylinders $K_{0}, \cdots, K_{n}$. Let $U_{j}^{\prime}$, $j=0,1, \cdots, n$, denote the interior of $K_{j}^{\prime}$ and let $M$ and $M^{\prime}$ denote, respectively, the closures of $U_{0}-\left(U_{1}+\cdots+U_{n}\right)$ and $U_{0}^{\prime}$ $-\left(U_{1}^{\prime}+\cdots+U_{n}^{\prime}\right)$. It follows from Theorem 1 and Lemma 2 of [4] and the remark following the proof of Theorem 5 of [3] that there is a homeomorphism $h$ of $M$ onto $M^{\prime}$ which does not change the $z$-coordinate of any point. For $j=0,1, \cdots, n$, let $G_{j}^{\prime}$ denote the collection of all images under $h$ of elements of $G_{j}$.

Let $C_{0}$ be a right circular cylinder with bases on $\alpha_{0}$ and $\alpha_{1}$ which is enclosed by $K_{0}^{\prime}$ and encloses each of $K_{1}^{\prime}, \cdots, K_{n}^{\prime}$. Let $C_{1}, \cdots, C_{n}$ be right circular cylinders which determine mutually exclusive solid cylinders, such that $C_{j}$ encloses $K_{j}^{\prime}$ and is enclosed by $C_{0}$. Let $V_{j}$ denote the interior of $C_{j}$, let $M_{0}=\mathrm{Cl}\left(U_{0}^{\prime}-V_{0}\right)$ and for $j=1,2, \cdots, n$, let $M_{j}=\mathrm{Cl}\left(V_{j}-U_{j}^{\prime}\right)$. It follows from Lemma 2 that, for $j=0,1, \cdots, n$, there exists a continuous collection $H_{j}$ of mutually exclusive arcs filling up $M_{j}$ such that no element of $H_{j}$ contains two points of any horizontal plane, $G_{j}^{\prime} \subset H_{i}$, and every element of $H_{j}$ which intersects $C_{j}$ is a vertical interval. Let $H=H_{1}+H_{2}+\cdots+H_{n}$ and let $G^{\prime}$ denote the collection obtained by adding to $H$ all vertical intervals with end points on $\alpha_{0}$ and $\alpha_{1}$ which intersect $M^{\prime}$. Then the collection $G$ of all images under $h^{-1}$ of elements of $G^{\prime}$ satisfies the desired conditions.

Theorem. Suppose $\alpha_{0}$ and $\alpha_{1}$ are horizontal planes and $G$ is a continuous collection of mutually exclusive arcs such that (1) each element of $G$ is irreducible from $\alpha_{0}$ to $\alpha_{1}$ and no element of $G$ contains two points of any horizontal plane, and (2) $G^{*}$ is compact and intersects $\alpha_{0}$ in a totally disconnected set. In order that there should exist a homeomorphism of $E^{3}$ onto itself which takes each element of $G$ onto a vertical interval and 
does not change the z-coordinate of any point, it is necessary and suffcient that for every positive number $\epsilon$, there exist a finite set $K_{1}, K_{2}, \cdots$, $K_{n}$ of topological cylinders with bases on $\alpha_{0}$ and $\alpha_{1}$ such that (1) the solid cylinders determined by $K_{1}, K_{2}, \cdots, K_{n}$ are mutually exclusive, (2) each arc of $G$ is enclosed by some $K_{i}$ and (3) each $K_{i}$ has horizontal diameter less than $\epsilon$.

Proof. 1. Suppose there is a homeomorphism $h$ of $E^{3}$ onto itself which takes each element of $G$ onto a vertical interval and does not change the $z$-coordinate of any point. Let $G^{\prime}$ denote the set of images under $h$ of the elements of $G$ and let $K^{\prime}$ be a vertical cylinder with bases on $\alpha_{0}$ and $\alpha_{1}$ which encloses $G^{*}$.

Suppose $\epsilon$ is a positive number. Let $S$ be a compact set containing the solid cylinder determined by $K^{\prime}$ in its interior. There is a positive number $\delta$ such that if $p$ and $q$ are points of $S$ and $\rho(p, q)<\delta$, then $\rho\left(h^{-1}(p), h^{-1}(q)\right)<\epsilon$. Since $\alpha_{0} \cdot G^{*}$ is compact and totally disconnected, there exists a finite set $D_{1}, D_{2}, \cdots, D_{n}$ of mutually exclusive disks in $\alpha_{0}$, each of diameter less than $\epsilon$, such that every point of $\alpha_{0} \cdot G^{*}$ is in the interior of some $D_{i}$. Let $K_{i}^{\prime}, i=1,2, \cdots, n$, denote the topological cylinder having $D_{i}$ as one of its bases and having its other base on $\alpha_{1}$, which has a collection of vertical intervals as a set of generators. If $K_{i}=h^{-1}\left(K_{i}^{\prime}\right)$, then $K_{1}, K_{2}, \cdots, K_{n}$ satisfy the conditions of the theorem.

2. Suppose the condition is satisfied. Let $K$ be a topological cylinder having a set of vertical generators, such that the bases of $K$ are on $\alpha_{0}$ and $\alpha_{1}$ and $K$ encloses $G^{*}$. By hypothesis, there exists a sequence $H_{1}, H_{2}, H_{3}, \cdots$ such that (1) for each $n, H_{n}$ is a finite collection of cylinders each having one base on $\alpha_{0}$ and the other on $\alpha_{1}$, such that no two of the solid cylinders determined by the elements of $H_{n}$ have a point in common, (2) $\mathrm{K}$ encloses each element of $H_{1}$ and for each $n$, each element of $H_{n+1}$ is enclosed by some element of $H_{n}$, (3) for each $n$, each arc of $G$ is enclosed by some element of $H_{n}$, and (4) for each $n$, each element of $H_{n}$ has horizontal diameter less than $1 / n$.

Let $U$ denote the interior of the closed cylinder determined by $K$ and for each $n$, let $U_{n}$ denote the sum of the interiors of the closed cylinders determined by the elements of $H_{n}$.

Let $G_{0}$ be the set of vertical generators for $K$. It follows from Lemma 3 that there exists a continuous collection $G_{1}$ of mutually exclusive arcs filling up the closure of $U-U_{1}$ such that (1) each element of $G_{1}$ is irreducible from $\alpha_{0}$ to $\alpha_{1}$ and no element of $G_{1}$ contains two points of any horizontal plane and (2) $G_{0} \subset G_{1}$ and each arc of $G_{1}$ which intersects an element of $H_{1}$ is a subset of that element. By 
applying Lemma 3 to each element of $H_{1}$, it can be shown that there is a continuous collection $G_{2}$ of mutually exclusive arcs filling up the closure of $U-U_{2}$, satisfying the first condition imposed on $G_{1}$ above and such that $G_{1} \subset G_{2}$ and each arc of $G_{2}$ which intersects an element of $\mathrm{H}_{2}$ is a subset of that element. By continuing this process, there may be obtained a sequence $G_{1}, G_{2}, G_{3}, \cdots$ such that (1) for each $n, G_{n}$ is a continuous collection of mutually exclusive arcs filling up the closure of $U-U_{n}$ such that each element of $G_{n}$ is irreducible from $\alpha_{0}$ to $\alpha_{1}$ and no element of $G_{n}$ contains two points of any horizontal plane, and (2) for each $n, G_{n} \subset G_{n+1}$. Let $G^{\prime}=G+G_{1}+G_{2}+\ldots$. Then $G^{\prime}$ is a continuous collection of mutually exclusive arcs filling up the solid cylinder determined by $K$, each element of $G^{\prime}$ is irreducible from $\alpha_{0}$ to $\alpha_{1}$, no element of $G^{\prime}$ contains two points of any horizontal plane, and each element of $G^{\prime}$ which intersects $K$ is a vertical interval.

Let $M$ denote the solid cylinder determined by $K$. For each point $p$ of $M$, let $f(p)$ be that point $q$ on the horizontal plane containing $p$ such that the projection of $q$ onto $\alpha_{0}$ is an end point of the arc of $G^{\prime}$ containing $p$. Then $f$ is a homeomorphism of $M$ onto itself which is fixed on $K$ and on $M \cdot \alpha_{0}$, does not change the $z$-coordinate of any point, and takes each element of $G^{\prime}$ onto a vertical interval. Let $F$ be the function which agrees with $f$ on $M$, leaves fixed each point of $E^{3}-M$ not lying directly above a point of $M$, and is such that if $p$ is a point of $E^{3}-M$ lying directly above the point $q$ of $\alpha_{1} \cdot M$ (supposing $\alpha_{1}$ is above $\alpha_{0}$ ), then $F(p)$ is the point with the same $z$-coordinate as $p$ which lies directly above the point $f(q)$. Then $F$ is a homeomorphism of $E^{3}$ onto itself which satisfies all the desired conditions.

\section{REFERENCES}

1. R. H. Bing, $A$ decomposition of $E^{3}$ into points and tame arcs such that the decomposition space is topologically different from $E^{3}$, Ann. of Math. vol. 65 (1957) pp. 484499.

2. U Upper semicontinuous decompositions of $E^{3}$, Ann. of Math. vol. 65 (1957) pp. 363-374.

3. E. Dyer and M.-E. Hamstrom, Completely regular mappings, Fund. Math. vol. 45 (1958) pp. 103-118.

4. M.-E. Hamstrom and E. Dyer, Regular mappings and the space of homeomorphisms on a 2-manifold, Duke Math. J. vol. 25 (1958) pp. 521-531.

5. M. K. Fort, Jr., A note concerning a decomposition space defined by Bing Ann. of Math. vol. 65 (1957) pp. 499-504.

UNIVERSITY OF VIRGINIA 